\title{
An Enrichment Scheme for Solidification Problems
}

Alejandro Cosimo Víctor Fachinotti Alberto Cardona

Centro Internacional de Métodos Numéricos Aplicados a la Ingeniería

(CIMEC-INTEC), Universidad Nacional del Litoral-CONICET

10th World Congress on Computational Mechanics

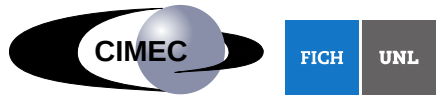




\section{Outline}

- Mathematical setting of the problem 


\section{Outline}

- Mathematical setting of the problem

- Numerical strategies to solve the problem 


\section{Outline}

- Mathematical setting of the problem

- Numerical strategies to solve the problem

- The proposed 1D solution 


\section{Outline}

- Mathematical setting of the problem

- Numerical strategies to solve the problem

- The proposed 1D solution

- Application examples 


\section{Outline}

- Mathematical setting of the problem

- Numerical strategies to solve the problem

- The proposed 1D solution

- Application examples

- Two dimensional extension 


\section{Outline}

- Mathematical setting of the problem

- Numerical strategies to solve the problem

- The proposed 1D solution

- Application examples

- Two dimensional extension

- Conclusions and observations 


\section{Mathematical setting}

Equation

$$
\rho \dot{\mathcal{H}}=Q+\nabla \cdot(k \nabla T)
$$

Initial Condition and boundary conditions

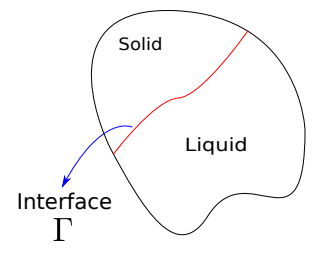

$$
\begin{aligned}
T & =T_{0} \\
T & =T_{d} \\
-(k \nabla T) \cdot \mathbf{n} & =q_{w} \\
-(k \nabla T) \cdot \mathbf{n} & =h_{f}\left(T-T_{f}\right)
\end{aligned}
$$

Constraints on the interface

$$
\begin{aligned}
T & =T_{m} \\
{[-(k \nabla T) \cdot \mathbf{n}]_{\Gamma} } & =\rho \mathcal{L} \mathbf{u}_{\Gamma}
\end{aligned}
$$

$\mathcal{L}$ : Latent Heat $\mathbf{u}_{\Gamma}$ : Velocity of $\Gamma$ $f_{l}$ : Liquid fraction (a Heaviside step)

c: Heat Capacity $\mathcal{H}$ : Specific Enthalpy $Q$ : Heat Source $\rho$ : density 


\section{Numerical strategies to solve the problem}

- Moving mesh or front tracking methods

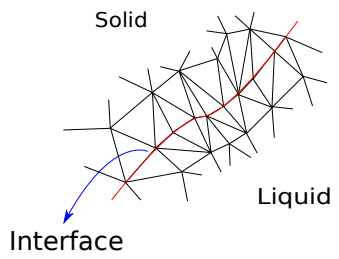




\section{Numerical strategies to solve the problem}

- Moving mesh or front tracking methods

- Fixed mesh methods

- Enthalpy Methods

- Capacitance Methods

- Temperature Based Methods

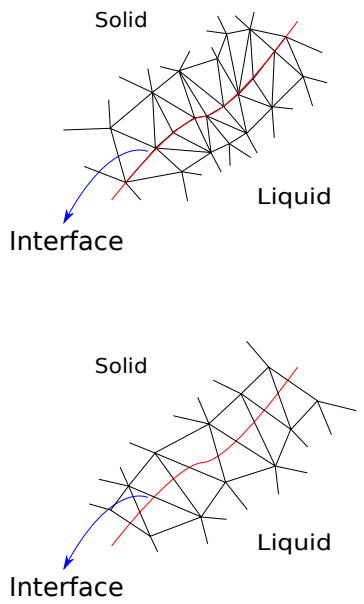




\section{Numerical strategies to solve the problem}

- Moving mesh or front tracking methods

- Fixed mesh methods

- Enthalpy Methods

- Capacitance Methods

- Temperature Based Methods

- Our Objective: enrich the space.

Literature: an auxiliary formulation to

evolve the interface is used

- Chessa, Smolinsky and Belytschko (2002)

- Ji, Chopp and Dolbow (2002)

- Merle and Dolbow (2002)

- Bernauer and Herzog (2011)

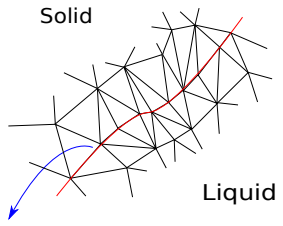

Interface

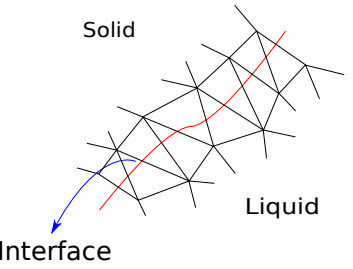




\section{Example: fixed mesh method without representing the gradient discontinuity}

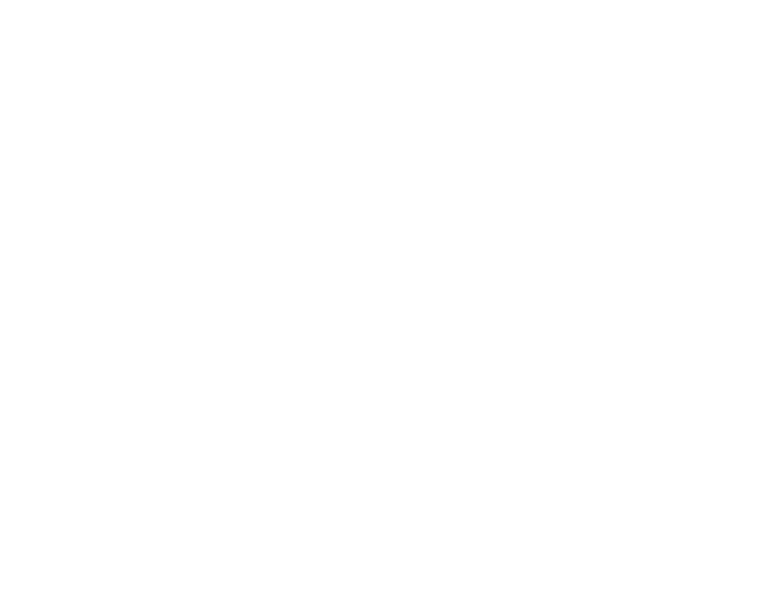




\section{The proposed Enrichment Function}

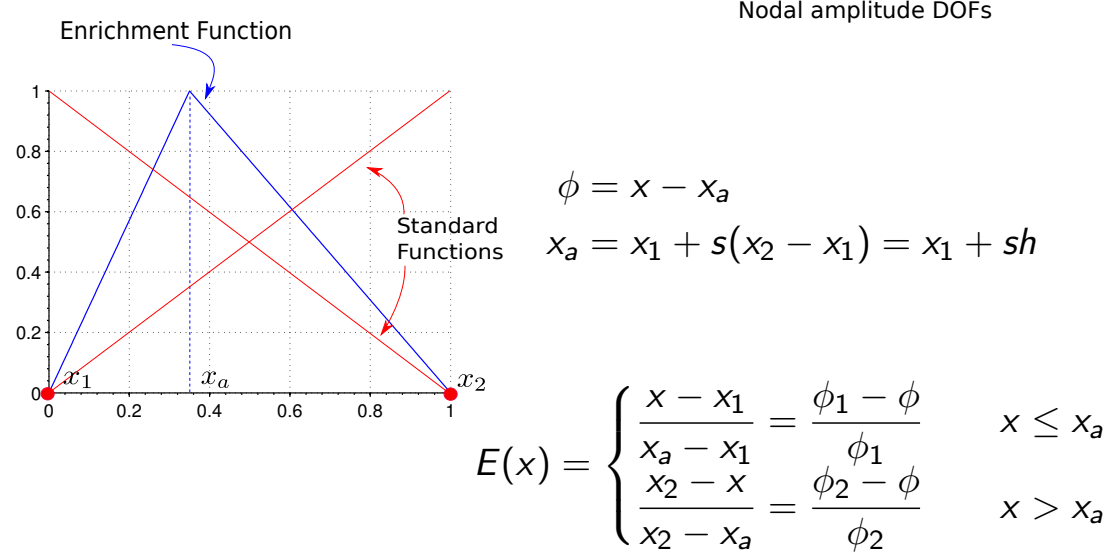




\section{Variational Formulation}

$$
\sum_{i \in[s, l]} \int_{\Omega_{i}} w[\rho \dot{\mathcal{H}}-\nabla \cdot(k \nabla T)-Q] d \Omega_{i}=0
$$

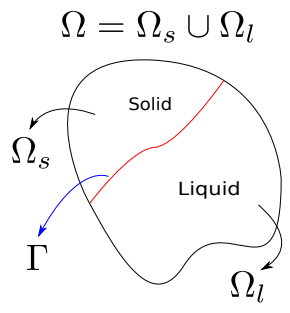




\section{Variational Formulation}

$$
\sum_{i \in[s, l]} \int_{\Omega_{i}} w[\rho \dot{\mathcal{H}}-\nabla \cdot(k \nabla T)-Q] d \Omega_{i}=0
$$

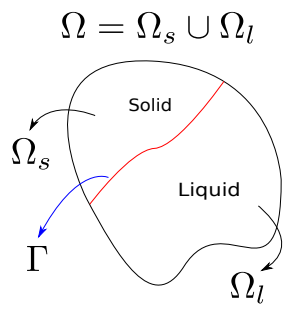

Then, making use of

- $\mathcal{H}=\mathcal{H}^{\text {sen }}+\mathcal{H}^{\text {lat }}=\int_{T_{\text {ref }}}^{T} c(\tau) d \tau+\mathcal{L} f_{l}(T)$

$\mathcal{L}$ : Latent Heat $f_{l}$ : Liquid fraction (a Heaviside step)

c: Heat Capacity $\mathcal{H}$ : Specific Enthalpy

$Q$ : Heat Source $\rho$ : density 


\section{Variational Formulation}

$$
\sum_{i \in[s, l]} \int_{\Omega_{i}} w[\rho \dot{\mathcal{H}}-\nabla \cdot(k \nabla T)-Q] d \Omega_{i}=0
$$

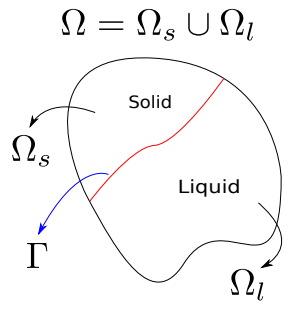

Then, making use of

- $\mathcal{H}=\mathcal{H}^{\text {sen }}+\mathcal{H}^{\text {lat }}=\int_{T_{\text {ref }}}^{T} c(\tau) d \tau+\mathcal{L} f_{l}(T)$

- The Reynolds Theorem

$\mathcal{L}$ : Latent Heat $f_{l}$ : Liquid fraction (a Heaviside step)

c: Heat Capacity $\mathcal{H}$ : Specific Enthalpy $Q$ : Heat Source $\rho$ : density 


\section{Variational Formulation}

Find $T \in \mathcal{S}$ such that $\forall w \in \mathcal{V}$

Term coming from $\dot{\mathcal{H}}$

$$
\int_{\Omega} w\left[\frac{\left.\nearrow_{\left[\rho \frac{\partial T}{\partial t}+\rho \mathcal{L} \frac{\partial f_{l}}{\partial t}\right]}-Q\right] d \Omega+\int_{\Omega} \nabla w \cdot(k \nabla T) d \Omega+}{C}\right.
$$

$$
\underbrace{\int_{\Gamma_{c}} w h_{f}\left(T-T_{f}\right) d \Gamma_{c}}_{\text {Robin BC }}+\int_{\text {Neumman BC }} w q_{w} d \Gamma_{q}=0
$$




\section{Time and Spatial Discretisations:} one dimensional case

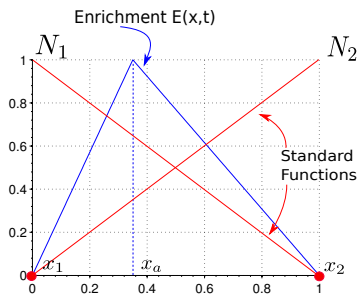

Taking

$$
T^{h}=\mathbf{N}^{T} \mathbf{T}
$$

where

$$
\mathbf{N}=\left[\begin{array}{c}
N_{1}(x) \\
N_{2}(x) \\
E(x, t)
\end{array}\right] \quad \text { and } \quad \mathbf{T}=\left[\begin{array}{c}
T_{1} \\
T_{2} \\
a
\end{array}\right]
$$




\section{Time and Spatial Discretisations}

We have

$$
\mathbf{\Pi}=\frac{\mathbf{C T}_{n+1}}{\Delta t}-\frac{\mathbf{C}^{*} \mathbf{T}_{n}}{\Delta t}+\frac{\mathbf{L}_{n+1}-\mathbf{L}_{n}}{\Delta t}+\mathbf{K} \mathbf{T}_{n+1}+\mathbf{F}-\mathbf{Q}
$$

where

$$
\begin{aligned}
\mathbf{C}^{*} & =\int_{\Omega} \rho c_{n+1} \mathbf{N}_{n+1} \mathbf{N}_{n}^{T} d \Omega & \\
\mathbf{L}_{n+1} & =\int_{\Omega} \rho \mathcal{L} \mathbf{N}_{n+1} f_{l(n+1)} d \Omega & \mathbf{L}_{n}=\int_{\Omega} \rho \mathcal{L} \mathbf{N}_{n+1} f_{l(n)} d \Omega
\end{aligned}
$$




\section{Interface Position Determination}

Phase Change Element

Basically we make use of the constraint

$$
\left.T\right|_{\Gamma}=T_{m}
$$

and using the introduced enrichment function, we have

$$
s=\frac{T_{m}-T_{1}^{(i)}-a^{(i)}}{T_{2}^{(i)}-T_{1}^{(i)}}
$$




\section{Discontinuous Integration}

We take as an example the term $\mathbf{C}^{*}$

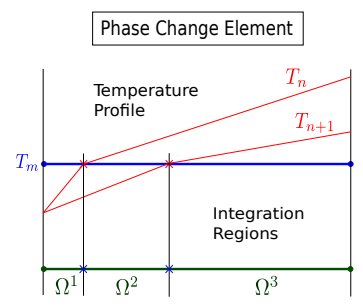

$$
\begin{aligned}
\mathbf{C}^{*}=\int_{\Omega} \rho c \mathbf{N}_{n+1} \mathbf{N}_{n}^{T} d \Omega & = \\
& \sum_{p=1}^{3} \sum_{g=1}^{n_{g}} \rho c \mathbf{N}_{n+1}\left(x_{g}^{(p)}\right) \mathbf{N}_{n}^{T}\left(x_{g}^{(p)}\right) w_{g} \Omega^{(p)}
\end{aligned}
$$




\section{Tangent Matrix}

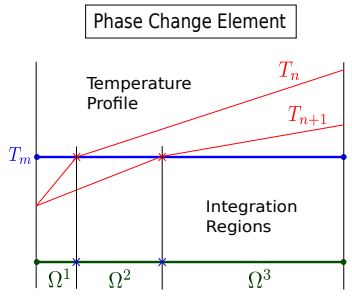

From the previous slide we can detect three sources where $\mathbf{C}^{*}$ depends on $\mathbf{T}$ :

- Evaluation dependency 


\section{Tangent Matrix}

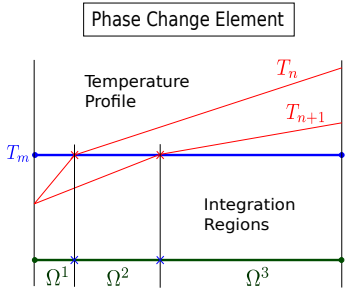

From the previous slide we can detect three sources where $\mathbf{C}^{*}$ depends on $\mathbf{T}$ :

- Evaluation dependency

- Enrichment definition dependency 


\section{Tangent Matrix}

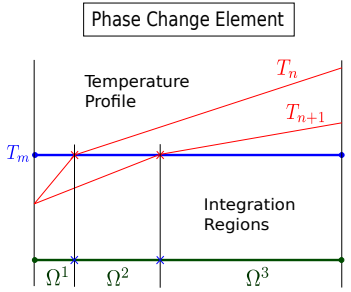

From the previous slide we can detect three sources where $\mathbf{C}^{*}$ depends on $\mathbf{T}$ :

- Evaluation dependency

- Enrichment definition dependency

- Integration region dependency 


\section{Tangent Matrix}

In the following $p$ represents the number of subdomains and $n_{g}$ the number of Gaussian points. To take an idea of the needed derivatives, take a glance to $\left(\frac{\partial \mathbf{C}^{*}}{\partial \mathbf{T}}\right)$ :

$$
\begin{aligned}
& \frac{\partial C_{r k}^{*}}{\partial T_{j}}=\sum_{p=1}^{3} \sum_{g=1}^{n_{g}} \rho c\left[\frac{\partial N_{n+1(r)}}{\partial x_{g}^{(p)}} \frac{\partial x_{g}^{(p)}}{\partial s} \frac{\partial s}{\partial T_{j}} N_{n(k)} w_{g} \Omega^{(p)}+\right. \\
& N_{n+1(r)} \frac{\partial N_{n(k)}}{\partial x_{g}^{(p)}} \frac{\partial x_{g}^{(p)}}{\partial s} \frac{\partial s}{\partial T_{j}} w_{g} \Omega^{(p)}+ \\
& \frac{\partial N_{n+1(r)}}{\partial x_{a}} \frac{\partial x_{a}}{\partial s} \frac{\partial s}{\partial T_{j}} N_{n(k)} w_{g} \Omega^{(p)}+ \\
& \left.N_{n+1(r)} N_{n(k)} w_{g} \frac{\partial \Omega^{(p)}}{\partial s} \frac{\partial s}{\partial T_{j}}\right]
\end{aligned}
$$




\section{Tangent Matrix}

In the following $p$ represents the number of subdomains and $n_{g}$ the number of Gaussian points. To take an idea of the needed derivatives, take a glance to $\left(\frac{\partial \mathbf{C}^{*}}{\partial \mathbf{T}}\right)$ :

$$
\begin{aligned}
& \frac{\partial C_{r k}^{*}}{\partial T_{j}}=\sum_{p=1}^{3} \sum_{g=1}^{n_{g}} \rho c\left[\frac{\partial N_{n+1(r)}}{\partial x_{g}^{(p)}} \frac{\partial x_{g}^{(p)}}{\partial s} \frac{\partial s}{\partial T_{j}} N_{n(k)} w_{g} \Omega^{(p)}+\right. \\
& N_{n+1(r)} \frac{\partial N_{n(k)}}{\partial x_{g}^{(p)}} \frac{\partial x_{g}^{(p)}}{\partial s} \frac{\partial s}{\partial T_{j}} w_{g} \Omega^{(p)}+ \\
& \frac{\partial N_{n+1(r)}}{\partial x_{a}} \frac{\partial x_{a}}{\partial s} \frac{\partial s}{\partial T_{j}} N_{n(k)} w_{g} \Omega^{(p)}+ \\
& \left.N_{n+1(r)} N_{n(k)} w_{g} \frac{\partial \Omega^{(p)}}{\partial s} \frac{\partial s}{\partial T_{j}}\right]
\end{aligned}
$$




\section{Tangent Matrix}

The core of the idea: the derivative $\frac{\partial s}{\partial T_{j}}$

After some computations, we have

$$
\frac{\partial s}{\partial \mathbf{T}}=-\left(\sum_{i=1}^{2} h \frac{\partial N_{i}}{\partial x}\left(x_{a}\right) T_{i}\right)^{-1}\left[\begin{array}{c}
N_{1}\left(x_{a}\right) \\
N_{2}\left(x_{a}\right) \\
1
\end{array}\right]
$$




\section{Algorithmic Implementation}

Some special treatment must be considered when one of following situations is detected:

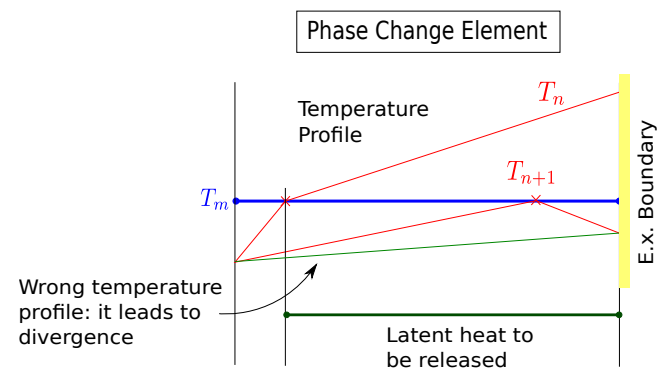

- Once an element is enriched and as the simulation evolves, careful attention must be paid to the elemental latent heat contribution in order to accurately determine the element state. 


\section{Algorithmic Implementation}

Some special treatment must be considered when one of following situations is detected:

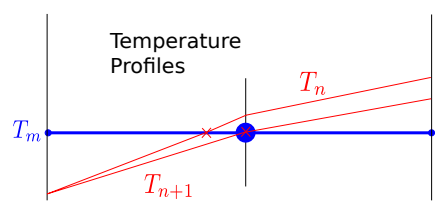

- If the parameter $s$ and the parameter associated to the enrichement $a$ are below or above certain thresholds, the element is not enriched. 


\section{Algorithmic Implementation}

Some special treatment must be considered when one of following situations is detected:

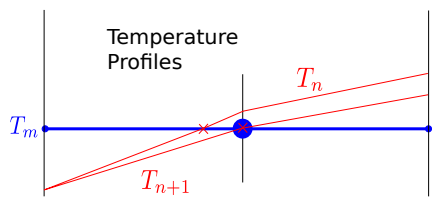

- If the parameter $s$ and the parameter associated to the enrichement $a$ are below or above certain thresholds, the element is not enriched.

- When the parameter $s$ is outside the range $(0,1)$, the element is considered liquid or solid depending on which state is most likely. 


\section{Application: Example I}

Example I: we study the frezzing of a long slab of length $L$ with two Dirichlet boundary conditions at its ends. Parameters of the problem:

$-10^{\circ} \mathrm{C}$

$$
\begin{gathered}
T_{m}=0^{\circ} \mathrm{C} \\
\mathcal{L}=190.26 \frac{\mathrm{J}}{\mathrm{kg}}
\end{gathered}
$$

$$
T_{0}=4^{\circ} \mathrm{C}
$$




\section{Application: Example I}

\section{With Enrichment}

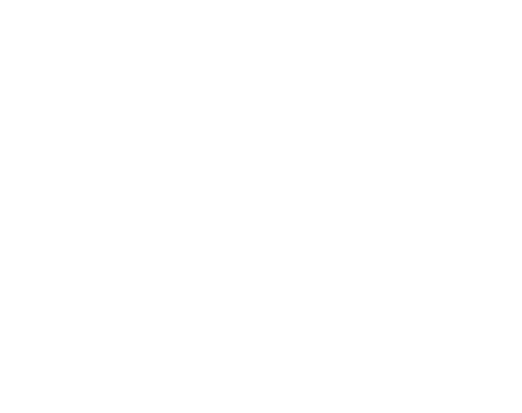

\section{Without Enrichment}

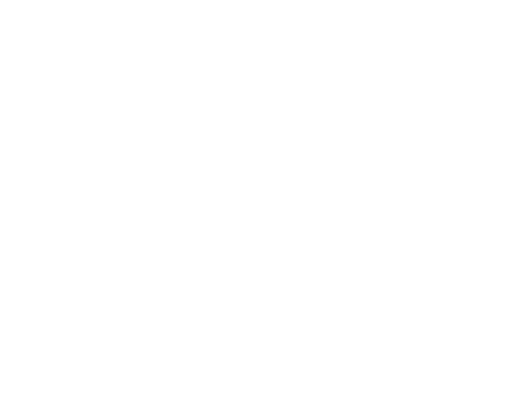




\section{Application: Example I}
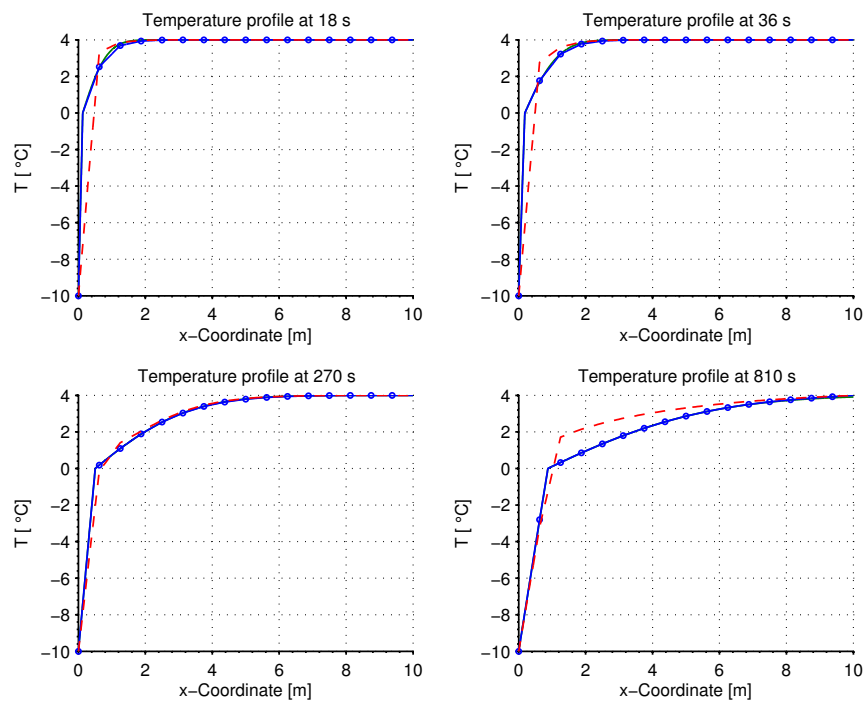


\section{Application: Example I}

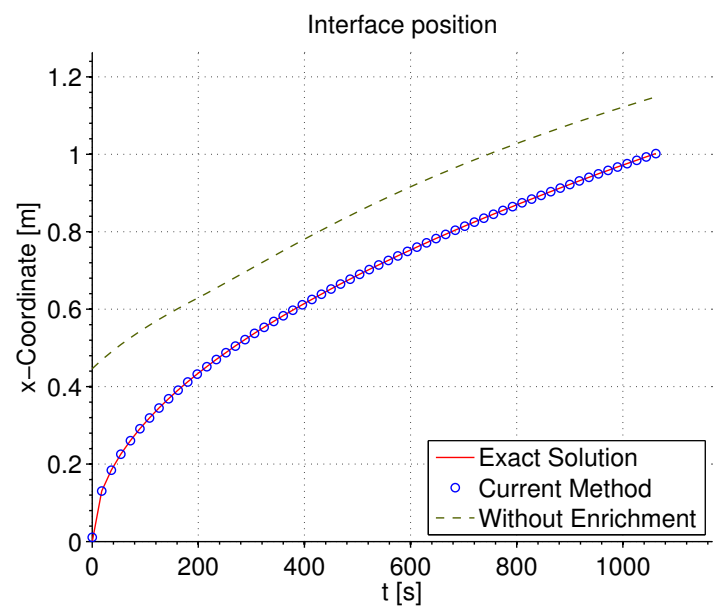




\section{Application: Example I}

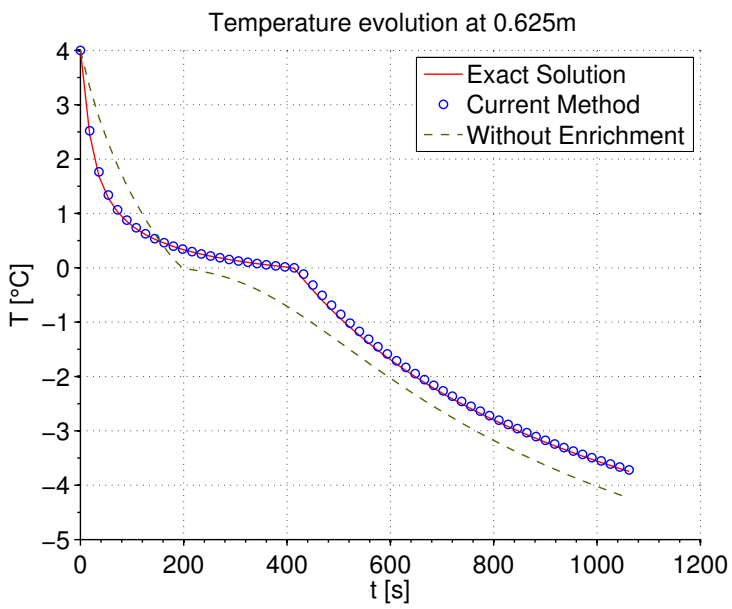




\section{Application: Example I}

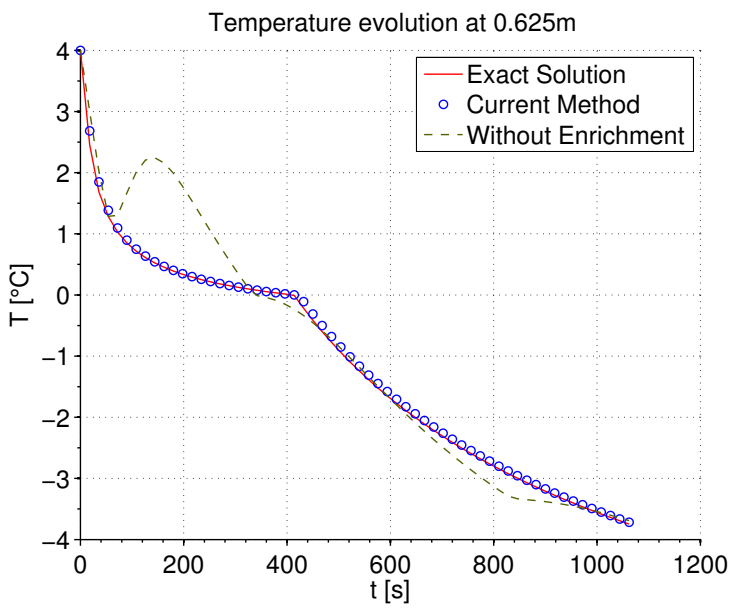




\section{Application: Example II}

Example II: we study the melting of a long slab of length $L$ with one Dirichlet condition and one Neumann boundary condition. Parameters of the problem:

$$
\begin{aligned}
& T_{m}=-0.1^{\circ} \mathrm{C} \\
& \mathcal{L}=190.26 \frac{\mathrm{J}}{\mathrm{kg}} \\
& 45^{\circ} \mathrm{C} \\
& 0 \frac{W K}{{ }^{\circ} \mathrm{Cm}^{2}} \\
& T_{0}=-1.1^{\circ} \mathrm{C}
\end{aligned}
$$




\section{Application: Example II}
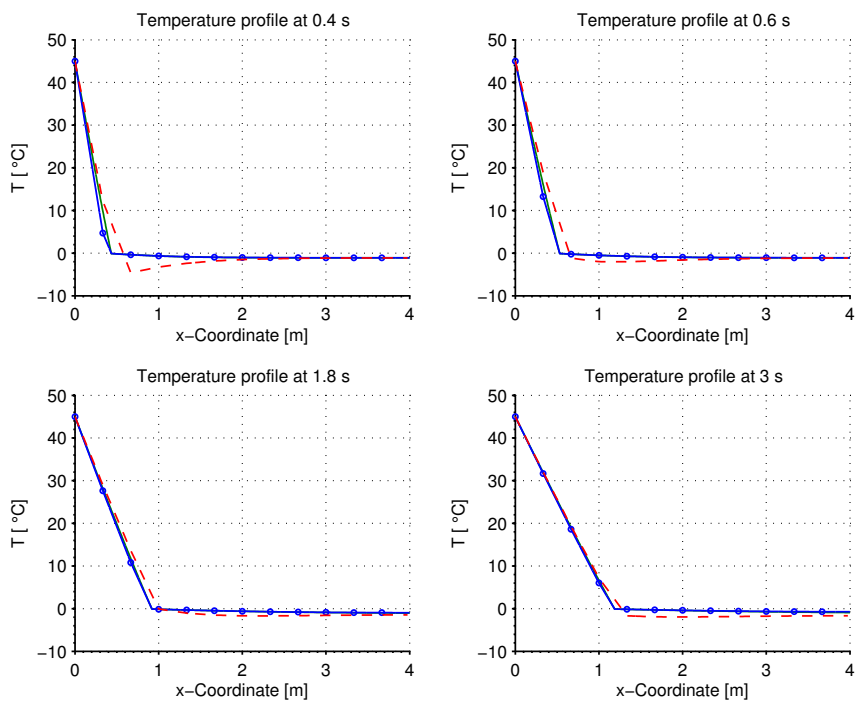


\section{Application: Example II}

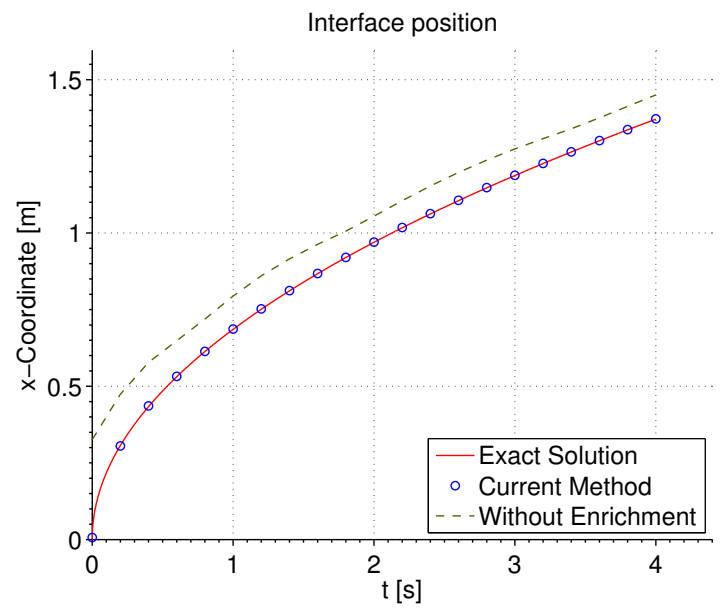




\section{Two Dimensional Extension}
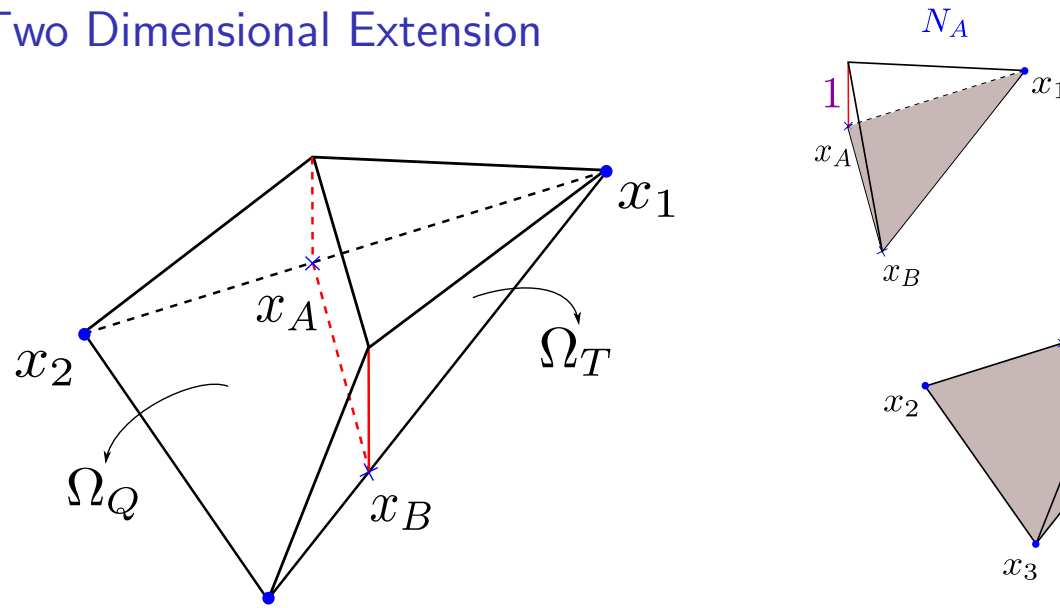

$$
x_{3}
$$$$
E(\mathbf{x})= \begin{cases}E_{Q}(\mathbf{x})=Q_{A}(\mathbf{x})+Q_{B}(\mathbf{x}) & \mathbf{x} \in \Omega_{Q} \\ E_{T}(\mathbf{x})=N_{A}(\mathbf{x})+N_{B}(\mathbf{x}) & \mathbf{x} \in \Omega_{T}\end{cases}
$$ 


\section{Conclusions and observations}

- Fixed domain method with the ability to represent the discontinuity in the temperature gradient inside the element. 


\section{Conclusions and observations}

- Fixed domain method with the ability to represent the discontinuity in the temperature gradient inside the element.

- No level set auxiliary formulation. 


\section{Conclusions and observations}

- Fixed domain method with the ability to represent the discontinuity in the temperature gradient inside the element.

- No level set auxiliary formulation.

- Accurate solutions were obtained. 


\section{Conclusions and observations}

- Fixed domain method with the ability to represent the discontinuity in the temperature gradient inside the element.

- No level set auxiliary formulation.

- Accurate solutions were obtained.

- The nonlinearity of the problem increases. 


\section{Conclusions and observations}

- Fixed domain method with the ability to represent the discontinuity in the temperature gradient inside the element.

- No level set auxiliary formulation.

- Accurate solutions were obtained.

- The nonlinearity of the problem increases.

- Besides, when the temperature profile is close to the melting temperature, we approach to the one phase problem. 


\section{Conclusions and observations}

- Fixed domain method with the ability to represent the discontinuity in the temperature gradient inside the element.

- No level set auxiliary formulation.

- Accurate solutions were obtained.

- The nonlinearity of the problem increases.

- Besides, when the temperature profile is close to the melting temperature, we approach to the one phase problem.

- Work in progress: two dimensional extension. 


\title{
Thanks for your attention
}

\author{
Questions?
}

